\section{Viskosität der Synovialflüssigkeit}

H.-D. Haubeck

Wasserburg, Deutschland

Synonym(e) Synovialflüssigkeits-Viskosität

Englischer Begriff viscosity

Definition Die Messung der Viskosität in der Synovialflüssigkeit erfolgt zur Differenzialdiagnose von entzündlichen und degenerativen Gelenkerkrankungen.

Beschreibung Die Viskosität einer Flüssigkeit ist eine Eigenschaft, die auf die innere Reibung, d. h. die Wechselwirkung von Molekülen der Flüssigkeit untereinander, zurückzuführen ist. Blut und Synovialflüssigkeit sind NichtNewtonsche Flüssigkeiten, d. h., die Viskosität ist von der Fließgeschwindigkeit abhängig.

Die Viskosität der Synovialflüssigkeit ist unter standardisierten Bedingungen $\left(25^{\circ} \mathrm{C}\right)$ im Wesentlichen abhängig von der Hyaluronankonzentration und der Molmassenverteilung des Hyaluronans (mittlere Molmasse 6-7 $\times 10^{6} \mathrm{Da}$ ). Bei entzündlichen Gelenkerkrankungen kommt es insbesondere durch die Wirkung von Sauerstoffradikalen, die von neutrophilen Granulozyten und Monozyten/Makrophagen freigesetzt werden, zur Depolymerisation des Hyaluronans und einer starken Abnahme der Viskosität. Bei der Osteoarthrose/arthritis findet sich dagegen eine normale oder erhöhte Viskosität.
Die Viskosität in der Synovialflüssigkeit lässt sich mit verschiedenen Verfahren messen ( $\downarrow$ Viskosimetrie). Neben den Kapillarviskosimetern nach Ostwald und Ubbelohde werden häufig Rotationsviskosimeter eingesetzt, bei denen ein Messkörper in der zu untersuchenden Substanz rotiert. Die Kalibration erfolgt mit Ölen definierter Viskosität.

Die Einheit (SI) der Viskosität ist die Pascal-Sekunde (Pas): Einheit $\eta=\mathrm{Pas}=\mathrm{Nsm}^{-2}=\mathrm{kg} / \mathrm{ms}$. Häufig findet sich noch die alte Angabe in Poise (P). $1 \mathrm{P}=0,1$ Pas.

Eine grobe Abschätzung der Viskosität lässt sich auch mit dem Mucin-Faden-Test gewinnen. Hierbei wird ein Tropfen Synovialflüssigkeit zwischen Daumen und Zeigefinger gegeben, beim Spreizen der Finger kommt es zur Fadenbildung. Die Länge des Fadens bis zum Reißen ist abhängig von der Viskosität. Der subjektive > Mucin-Clot-Test, bei dem durch Zugabe von Essigsäure das $>$ Hyaluronan gefällt wird und das Aussehen des sog. Mucin-Clots bewertet wird, sollte nicht mehr verwendet werden.

\section{Literatur}

Dahl LB, Dahl IMS, Engström-Laurent A et al (1985) Concentration and molecular weight of sodium hyaluronate in synovial fluid from patients with rheumatoid arthritis and other arthropathies. Ann Rheum Dis 44:817-822

Mc Cord JM (1974) Free radicals and inflammation: protection of synovial fluid by superoxide dismutase. Science 185:529-531

Stuhlsatz HW, Eberhard A, Kristin H et al (1976) Glycosaminoglycans in the synovial fluid of chronic joint diseases. Verh Dtsch Ges Rheumatol 4:444-454 